\title{
Cerebral Hypoxia
}

National Institute of Neurological Disorders and Stroke (NINDS)

\section{Source}

National Institute of Neurological Disorders and Stroke (NINDS). Cerebral Hypoxia

Information Page.

Cerebral hypoxia refers to a condition in which there is a decrease of oxygen supply to the brain even though there is adequate blood flow. Drowning, strangling, choking, suffocation, cardiac arrest, head trauma, carbon monoxide poisoning, and complications of general anesthesia can create conditions that can lead to cerebral hypoxia. Symptoms of mild cerebral hypoxia include inattentiveness, poor judgment, memory loss, and a decrease in motor coordination. Brain cells are extremely sensitive to oxygen deprivation and can begin to die within five minutes after oxygen supply has been cut off. When hypoxia lasts for longer periods of time, it can cause coma, seizures, and even brain death. In brain death, there is no measurable activity in the brain, although cardiovascular function is preserved. Life support is required for respiration. 